\title{
Stage III Laryngeal Cancer AJCC v8
}

National Cancer Institute

\section{Source}

National Cancer Institute. Stage III Laryngeal Cancer A/CC v8. NCI Thesaurus. Code C133160.

Stage III includes: (T3, N0, M0); (T1, N1, M0); (T2, N1, M0); (T3, N1, M0). T3:

Supraglottis: T umor is limited to larynx with vocal cord fixation and/or invades any of the following: postcricoid area, preepiglottic space, paraglottic space, and/or inner cortex of thyroid cartilage. Glottis: T umor is limited to the larynx with vocal cord fixation and /or invades the paraglottic space, and/or inner cortex of the thyroid cartilage. Subg lottis: Tumor is limited to the larynx with vocal cord fixation and/or invades the parag lottic space and/or inner cortex of the thyroid cartilage. T1: Suprag lottis: T umor is limited to one subsite of supraglottis with normal vocal cord mobility. Glottis: Tumor is limited to the vocal cord(s) (may involve anterior or posterior commissure) with normal mobility. Subglottis: Tumor is limited to the subg lottis. T2: Supraglottis: Tumor invades the mucosa of more than one adjacent subsite of supraglottis or glottis or region outside the suprag lottis (e.g., mucosa of base of the tongue, vallecula, medial wall of pyriform sinus) without fixation of the larynx. Glottis: Tumor extends to supraglottis and/or subglottis, and/or with impaired vocal cord mobility. Subglottis: Tumor extends to vocal cord(s) with normal or impaired mobility. N0: No regional lymph node metastasis. N1: Metastasis in a single ipsilateral lymph node, $3 \mathrm{~cm}$ or less in greatest dimension and ENE(-). M0: No distant metastasis. (AJCC 8th ed.) 